\title{
VERTICAL COMPACTION IN A FAULTED SEDIMENTARY BASIN
}

\author{
Gérard Gagneux ${ }^{1}$, Roland Masson $^{2}$, Anne Plouvier-Debaigt ${ }^{1}$, Guy Vallet $^{1}$ \\ AND SYLVIE WOLF ${ }^{2}$
}

\begin{abstract}
In this paper, we consider a 2D mathematical modelling of the vertical compaction effect in a water saturated sedimentary basin. This model is described by the usual conservation laws, Darcy's law, the porosity as a function of the vertical component of the effective stress and the Kozeny-Carman tensor, taking into account fracturation effects. This model leads to study the time discretization of a nonlinear system of partial differential equations. The existence is obtained by a fixed-point argument. The uniqueness proof, by Holmgren's method, leads to work out a linear, strongly coupled, system of partial differential equations and boundary conditions.
\end{abstract}

Mathematics Subject Classification. 35Q35, 76S05, 35J65.

Received: July 10, 2002. Revised: January 22, 2003.

\section{INTRODUCTION}

Extracted of [15]: "The constitution of a sedimentary basin during the geologic history implies processes of: sedimentation, erosion, compaction, eviction and transfer of fluids, thermic transfer and of diagenesis, of which outcome is a geologic structure capable of establishing a reservoir of hydrocarbons or a deposit of mineral resources." The modelling, at a geological scale, of these various mechanisms and their numerical simulations establish a promising tool for the evaluation of the oil potential of basins (see $[5,23]$ and $[24]$ ).

The simulation of the genesis and the migration of hydrocarbons in the sedimentary coverage has to take into account sedimentation and erosion phenomena, and so compaction of sediments. From then on, it is necessary to consider poromechanical models.

The reader interested in similar problems, outside the framework of hydrocarbons, will be able to consult Sciarra et al. [25] who consider a binary mixture where a dilatation of pores is observed under extremal pressure. One can also see the importance of compaction in the dynamics of large ice masses as mentioned by Gödert et al. in [13].

In this first approach, we shall suppose that the mechanics of cliffs (sediments) deformation ensues from the vertical compaction. The other phenomena of deformation, such as the gliding of the sedimentary layers for example, will be untidy, either, supposed known in advance and implicitly contained in the data of the problem. It will be enough then to consider a rheological model allowing to express the porosity variations according to

\footnotetext{
Keywords and phrases. Porous media, vertical compaction, sedimentary basins, fault lines modelling.

1 Laboratoire de Mathématiques Appliquées, Université de Pau et des Pays de l'Adour, BP 576, 64012 Pau Cedex, France.

e-mail: gerard.gagneux@univ-pau.fr, guy.vallet@univ-pau.fr

2 Institut Français du Pétrole, 1 et 4 avenue de Bois-Préau, BP 311, 92852 Rueil-Malmaison Cedex, France.

e-mail: roland.masson@ifp.fr
} 


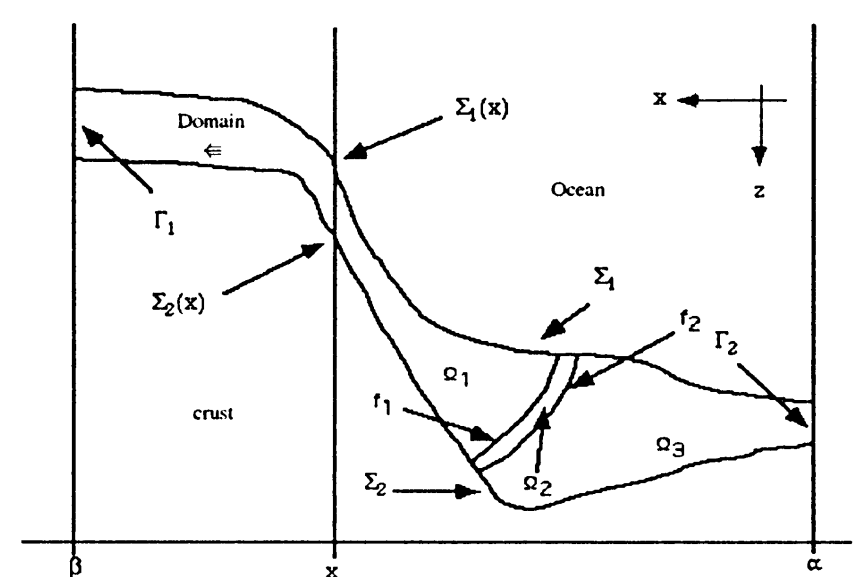

FiguRE 1. Faulted domain.

the only vertical constraint. This simplifying working hypothesis does not remove anything in the capability of the model as it is noticed by Luo et al. [17], Perez [22] or Wangen et al. [26] and [27].

The current study concerns a $2 \mathrm{D}$ monophasic model in a faulted porous medium. It takes into account the vertical compaction and the fracturing according to the state of the effective constraint, with an effect of threshold of release.

There are few mathematical publications on compaction models in sedimentary basins. Most of them concerns numerical aspects (see for example Badea [2], Fowler et al. [8], Ismail-zade et al. [14], Wangen [26], Wangen et al. [27] or Zakarian et al. [29]).

Our goal is to give some mathematical tools in order to analyse such models. Numerical aspects are actually studied at the Institut Français de Pétrole (Schneider et al. [23, 24]).

\section{Mathematical modelling}

As mentioned in the previous section, the anisotropy is mainly vertical, then a $2 \mathrm{D}$ model is considered in the plane $O x y$ with origin $O$, horizontal axis $x$ and vertical axis $z$ pointing to the direction of gravity. In this theoretical study, we consider that the physical domain $\Omega$ is decomposed into three sub-domains (cf. Fig. 1): sub-domains $\Omega_{1}$ and $\Omega_{3}$ represent two parts of a sedimentary basin $\Omega$, separated by a fault $\Omega_{2}$. The thickness of this fault is small compared with the size of $\Omega_{1}$ and $\Omega_{3}$, but it is not unimportant compared with the physical phenomena which it generates. So, we shall consider $\Omega_{2}$ as a separate sub-domain with appropriate characteristics, i.e. allowing a free passage in compulsory directions or causing obstructions.

This working hypothesis becomes delicate in numerical analysis, where it can be preferable to model this fault as an interface (so without thickness). From then on, this has to lead to artificial boundary conditions in order to restore the real role of this fault in the water - sediments traffic. One finds then, in previous studies, different subtleties.

In his approach, [22] imposes a continuance of the stream through the fault and introduces a parameter $\delta$ to take into account the discontinuity of the pressure between the superior wall and the lower wall. This discontinuity is necessary, otherwise, it would mean that this fault, with a null Lebesgue measure, is neglected in the system of equations and so would not exist physically.

As indicated by the author, the problem is that the determination of $\delta$ is purely empirical. Furthermore, this internal discontinuity prevents the representative function of the water pressure from being in a Sobolev's space of first order on $\Omega$, that does not receive any convincing explanations.

In an other approach, one imposes the continuity of the traces on both sides of the fault. But then, as mentioned above, the system of equations alters the physical reality. Then, in the modelling of the fault, 
one has to use a term of order 1 , and one may have jumps across the interface. It gives, in the formulation of equations, a supplementary term: a measure with support in the interface-fault, as if it was a well. Furthermore, it does not seem realistic that the pressure is the same on both sides of the fault.

In our approach, we adopt the principles of continuity of traces and of fluxes to write the relations which govern the water pressure in each interface. So, we take into account the physical process in $\Omega_{2}$ and jumps exist from an extreme edge to the other.

If for numerical motivations, one has to consider that the fault is an interface, a last approach could be based on an asymptotic analysis with regard to $\varepsilon$ (the parameter of the fault thickness) in order to find one of the above mentioned configurations.

The above proposed method, which represents a simplified model (one fault which cuts in two the studied domain) can be generalized to a sedimentary basin with several faults and several types of sediments. The main consideration is to be able to decompose the domain in a certain number of sub-domains with known geologic, rheologic, ..., characteristics.

In a first part, we are interested in the mathematical analysis of the model, where compactions are of weak amplitude. We study then the time semi-discretisation of the system. The existence of a solution results from Schauder-Tychonoff's fixed point theorem, in the separable hilbertian framework. Then, uniqueness is proved with a technique of transposition, inspired by Antontsev and Domansky's works [1] on the analytical study of diphasic filtration system. This technique, generalizing the method of pivot space changing [16], classically returns the study of the uniqueness of the primal problem to the study of the existence for the dual problem. This last one admits at least a solution due to Lax-Milgram's theorem.

In a second part, we consider a model where compaction is of general amplitude. To study this problem of predictive-corrective type, we do not make reference to the narrow-mindedness of certain coefficients in the state laws. Uniqueness is obtained by applying Fredholm's alternative, associated to a weak maximum principle.

\subsection{Notations}

Let $\alpha \in\{s, w\}$ ( $w$ label for water and $s$ label for sediments) and $i \in\{1,2,3\}$ (for domain $\Omega_{i}$ ):

$\vec{V}_{\alpha}:$ speed of phase $\alpha$,

$P_{\text {atm }}$ : atmospheric pressure,

$\rho_{\alpha}$ : volumic mass, $\quad p_{w}:$ water presure,

$\lambda_{a}^{i}$ and $\lambda_{s}^{i}$ : coefficients of anisotropy,

$S_{0}^{i}$ : specific area of the porous media,

$K_{i}$ : permeability tensor, $\quad \sigma_{z}$ : total constraint,

$k_{i}$ : absolute permeability, $\sigma$ : effective constraint,

$\phi_{r}^{i}, \phi_{a}^{i}, \phi_{b}^{i}, \sigma_{a}^{i}, \sigma_{b}^{i}$ : being characteristic parameters of the deposited sediment nature,

$g_{s}$ : sedimentation speed at the bottom of the ocean, $g$ : gravity acceleration, $H_{w}$ : height of water, $F_{i}=\left[\rho_{w} \phi_{i}+\rho_{s}\left(1-\phi_{i}\right)\right] g, \quad{ }^{t} \vec{B}=\left(0, \rho_{w} g\right)$.

\subsection{Conservation laws}

In each sub-domain $\Omega_{i}$, the conservation laws are the following ones:

1) mass conservation of the sediment and water:

$$
\begin{aligned}
& \frac{\partial}{\partial t}\left(\rho_{s}^{i}\left(1-\phi_{i}\right)\right)+\operatorname{div}\left(\rho_{s}^{i}\left(1-\phi_{i}\right) \vec{V}_{s}\right)=0, \\
& \frac{\partial}{\partial t}\left(\rho_{w} \phi_{i}\right)+\operatorname{div}\left(\rho_{w} \phi_{i} \vec{V}_{w}\right)=0 .
\end{aligned}
$$

2) momentum conservation (equilibrium equation)

$$
\frac{\partial \sigma_{z}}{\partial z}=\left(\phi_{i} \rho_{w}+\left(1-\phi_{i}\right) \rho_{s}^{i}\right) g=F_{i}\left(\phi_{i}\right) .
$$

One supposes in the sequel that $\rho_{s}$ and $\rho_{w}$ are constant on each sub-domain. 


\subsection{Behavior laws}

These conservation laws have to be completed by phenomenologic behavior laws. We held:

$$
\begin{array}{ll}
\phi_{i}\left(\vec{V}_{w}-\vec{V}_{s}\right)=-K_{i}\left(\overrightarrow{\nabla p}_{w}-\vec{B}\right) & \text { (Darcy's law), } \\
\sigma=\sigma_{z}-p_{w} & \text { (Terzaghi's relation) } \\
\phi_{i}(\sigma)=\phi_{r}^{i}+\phi_{a}^{i} \exp \left[-\frac{\sigma}{\sigma_{a}^{i}}\right]+\phi_{b}^{i} \exp \left[-\frac{\sigma}{\sigma_{b}^{i}}\right] & \text { (Elastoplastic rheological law), } \\
{ }^{t} \vec{V}_{s}=\left(0, v_{s}\right) & \text { (Vertical compaction hypothesis), } \\
K_{i}\left(\sigma, p_{w}\right)=k_{i}\left(\phi_{i}\right)\left(\begin{array}{ll}
\lambda_{s}^{i} & 0 \\
0 & \lambda_{a}^{i}
\end{array}\right)+\left(\begin{array}{ll}
0 & 0 \\
0 & h_{i}\left(\sigma, p_{w}\right)
\end{array}\right) & \text { (Permeability law) }
\end{array}
$$

where the tensor, expressed in a strate - antistrate base, rests on Kozeny-Carman's law with $k_{i}(\phi)=\frac{0,2 \phi_{i}^{3}}{S_{0}^{i 2}\left(1-\phi_{i}\right)^{2}}$. It is completed with the consideration of the fracturation, by means of a supplementary term $h_{i}$, which intervenes in the physical phenomenon as soon as certain critical threshold is reached.

\subsection{Description of the domain and of the boundary conditions}

- One supposes (geometrical regularity) that there are four Lipschitzian functions $\gamma_{1}, \gamma_{2}, f_{1}$ and $f_{2}$, such that (cf. Fig. 1):

$$
\begin{array}{ll}
\Omega=\left\{(x, z) \in \mathbb{R}^{2}, \alpha<x<\beta, \gamma_{1}(x)<z<\gamma_{2}(x)\right\}, & \Omega_{1}=\left\{(x, z) \in \Omega, z>f_{1}(x)\right\} \\
\Omega_{2}=\left\{(x, z) \in \Omega, f_{1}(x)>z>f_{2}(x)\right\}, & \Omega_{3}=\left\{(x, z) \in \Omega, z<f_{2}(x)\right\} \\
\Gamma_{1}=\left\{(\alpha, z), \gamma_{1}(\alpha)<z<\gamma_{2}(\alpha)\right\}, & \Gamma_{2}=\left\{(\beta, z), \gamma_{1}(\beta)<z<\gamma_{2}(\beta)\right\} \\
\Sigma_{i}=\left\{(x, z) \in \mathbb{R}^{2}, \alpha<x<\beta, z=\gamma_{i}(x)\right\}, & f_{i}=\left\{(x, z) \in \Omega, z=f_{i}(x)\right\}, i \in\{1,2\} .
\end{array}
$$

- Conditions on $\Sigma_{1}: p_{w}=\sigma_{z}=P_{\text {atm }}+\rho_{w} g H_{w} \underset{\text { notation }}{=} P_{\Sigma_{1}}$, in particular, according to (5), one has

$$
p_{w}=P_{\Sigma_{1}}, \quad \sigma=0 .
$$

In fact, Domain $\Omega$ evolves during time because of the erosion and of the sedimentation by gravitation on the free boundary $\Sigma_{1}$ (bottom of the ocean), according to the law: $v_{s}=g_{s}$ where, for example, $g_{s}=\frac{\overrightarrow{Q_{s}} \cdot \vec{n}}{n_{z}}, \overrightarrow{Q_{s}}$ ensuing from the sedimentary load of the ocean, the direction, the intensity of maritime currents...

- Condition on $\Sigma_{2}: \overrightarrow{V_{w}} \cdot \vec{n}=0$ and, according to (4), one gets

$$
-{ }^{t} \vec{n} K\left(\overrightarrow{\nabla p_{w}}-\vec{B}\right)=-\phi(\sigma) v_{s} n_{z}
$$

where $n_{z}$ represents the vertical part of the normal vector $\vec{n}$.

Furthermore, Domain $\Omega$ evolves, a priori, also during time by the motion of crusts. So $\Sigma_{2}$ is also a free boundary. We shall suppose it fixed in this study, in order to seriate the difficulties.

- Conditions on $\Gamma_{1}$ and $\Gamma_{2}$ (artificial free boundaries): $\overrightarrow{V_{w}} \cdot \vec{n}=0$. In particular, according to (4) and by noticing that ${ }^{t} \vec{n}=(+/-1,0), \overrightarrow{V_{s}} \cdot \vec{n}=0$, so that one has

$$
-{ }^{t} \vec{n} K\left(\overrightarrow{\nabla p_{w}}-\vec{B}\right)=0
$$


- Conditions in the internal interfaces of the domain $f_{i}, i=1,2$ :

$$
\left\{\begin{array}{c}
\sigma_{\Omega_{i}}=\sigma_{\Omega_{i+1}}, \quad v_{s, \Omega_{i}}=v_{s, \Omega_{i+1}}, \quad p_{w, \Omega_{i}}=p_{w, \Omega_{i+1}}, \\
-{ }^{t} \vec{n} K_{i}\left(\overrightarrow{\nabla p}_{w, \Omega_{i}}-\vec{B}\right)=-{ }^{t} \vec{n} K_{i+1}\left(\overrightarrow{\nabla p}_{w, \Omega_{i+1}}-\vec{B}\right) .
\end{array}\right.
$$

- Initial conditions (for $t=0$, there are no sediments):

$$
\Sigma_{1}=\Sigma_{2}, \quad \phi_{i}=\phi_{r}^{i}+\phi_{a}^{i}+\phi_{b}^{i}, \quad \sigma_{z}=p_{w}=P_{a t m}+\rho_{w} g H_{w}, \text { so } \sigma=0 .
$$

\subsection{Presentation of the system of equations}

First, we introduce some notations: Considering $\mathbb{I}_{\Omega_{i}}$ the caracteristic function of Domain $\Omega_{i}$, one sets,

$$
K(x, z, \sigma, p)=\sum_{i} K_{i}(\sigma, p) \mathbb{I}_{\Omega_{i}}(x, z), \quad \phi(x, z, \sigma)=\sum_{i} \phi_{i}(x, z, \sigma) \mathbb{I}_{\Omega_{i}}(x, z), \quad F(x, z, \sigma)=\sum_{i} F_{i}(\sigma) \mathbb{I}_{\Omega_{i}}(x, z)
$$

It is important to notice that each of these functions is regular on $\Omega_{i}$ and that it has a trace on $\partial \Omega_{i}$ (thus on $\Sigma_{i}$, $\Gamma_{i}$ and $f_{i}$ ).

A triple preliminary analysis of the partial differential equations introduced by the model, of the behavior laws which structure them and of the boundary and interfaces conditions imposed by the experimentation, leads to the following choice for the main unknown

$$
\sigma, v_{s} \text { and } p_{w}
$$

and to the following choice for the system of equations on $\Omega$

$$
\begin{aligned}
& \frac{\partial \sigma}{\partial z}=F(., ., \sigma)-\frac{\partial p_{w}}{\partial z} \\
& -\frac{\partial}{\partial t} \phi(., ., \sigma)+\frac{\partial}{\partial z}(1-\phi(., ., \sigma)) v_{s}=0 \\
& -\operatorname{div}\left\{K\left(., ., \sigma, p_{w}\right)\left(\overrightarrow{\nabla p_{w}}-\vec{B}\right)\right\}+\frac{\partial}{\partial z} v_{s}=0 .
\end{aligned}
$$

Equation (13) comes from the equilibrium equation (3) and Terzaghi's relation (5), equation (14) comes from the mass conservation of the sediment (1) and equation (15) comes from the mass conservation laws (1) and (2) and Darcy's law (4).

In the sequel, the analysis concerns the study of a time discretisation, reasonable method for the approach of slow evolution processes.

We do not plan to study the time continuous system. Indeed, contribution of sedimentation and erosion effects prevent a good control of the evolution of $\Omega$ during time, in order to pass easily to the limit. Here, the hypothesis of monotonicity for example of the application $t \mapsto \Omega(t)$, according to the ideas of [16] (p. 415), is not justifiable. However, we can propose a correction of Domain $\Omega$ at each iteration. Indeed, boundary $\Sigma_{1}$ is a free boundary, subjected to the oceanic phenomena of deposits or erosion.

Referring to a model which authorizes only vertical deformations, one transcribes the principle of the conservation of sedimentary material quantity on the vertical line, over the point $x$ of $\Sigma_{2}$, by

$$
\int_{\Sigma_{2}(x)}^{z^{*}(x)}\left(1-\widetilde{\phi}(., ., \sigma(x, z)) \mathrm{d} z=\int_{\Sigma_{2}(x)}^{\Sigma_{1}(x)}\left(1-\phi\left(., ., \sigma_{0}(x, z)\right) \mathrm{d} z+\left(\vec{Q}_{s} \cdot \vec{n}\right)_{x} . h\right.\right.
$$


where: $\left(\overrightarrow{Q_{s}} \cdot \vec{n}\right)_{x}$ represents the contribution or the volumic loss via the ocean, $z^{*}(x)$ is the new quotation for the boundary $\Sigma_{1}$ at the top of the point $x$ of $\Sigma_{2}$, supposed indeformable

$$
\widetilde{\phi}(., ., \sigma(x, z))=\phi(., ., \sigma(x, z)) \quad \text { if } \quad \mathrm{z} \leq \Sigma_{1}(\mathrm{x}), \quad \phi(., ., 0) \quad \text { if } \quad \mathrm{z} \geq \Sigma_{1}(\mathrm{x}) .
$$

Function $h_{x}$ (for any $x$ ) which gives, for any $z^{*}, \int_{\Sigma_{2}(x)}^{z^{*}}\left(1-\widetilde{\phi}(., ., \sigma(x, z)) \mathrm{d} z\right.$, is increasing over $\mathbb{R}^{+}$. For any $x$, this equation defines $z^{*}(x)$ in a unique way. So, it supplies the Cartesian equation of the new profile of $\Sigma_{1}$.

By keeping in memory the value of the maximal constraint punctually reached [22], let us notice that this process of rectification can be enriched with the irreversibility consideration of the compaction.

In the next section, we present the mathematical analysis of the first iteration of $(13,14)$ and $(15)$ in the case of an implicit discretisation. Moreover, weak amplitude compaction (i.e. with a small variation of $\phi$ and $K$ with respect to the unknown $\sigma$ and $p_{w}$ ) is considered.

In Section 4, we present the mathematical analysis of the first iteration of $(13,14)$ and $(15)$ in the case of a predictive-corrective type discretisation. General amplitude compaction is considered.

\section{Mathematical ANALysis of A FiRst CASE: WEAK AMPLitUde COMPACTION}

Let $h=\Delta t$ be the iteration step and $\sigma_{0}$ be the datum of $\sigma$ at the previous iteration. The proposed discretised scheme is based on equations (13) and (15) for the unknown $\sigma$ and $p_{w}$ and on

$$
\frac{\phi\left(., ., \sigma_{0}\right)-\phi(., ., \sigma)}{h}+\frac{\partial}{\partial z}\left((1-\phi(., ., \sigma)) v_{s}\right)=0,
$$

for the unknown $v_{s}$.

\subsection{Notations of functional analysis}

Then, one has to look for the solutions in an adapted cartesian product of first order Sobolev's spaces. In order to do so, one denotes

$$
V^{f}=\left\{u \in H^{1}(\Omega), u_{\mid \Sigma_{1}}=f\right\},
$$

where $f$ is given on $\Sigma_{1}$, regular enough so that $V^{f}$ is not empty.

$$
W=\left\{u \in L^{2}(\Omega), \frac{\partial u}{\partial z} \in L^{2}(\Omega)\right\}=L^{2}\left(\alpha<x<\beta, H^{1}\left[\gamma_{1}(x), \gamma_{2}(x)\right]\right) .
$$

$W$ is a separable Hilbert space for its natural norm

$$
\forall u \in W,\|u\|_{W}^{2}=\int_{\Omega} u^{2} \mathrm{~d} x+\int_{\Omega}\left(\frac{\partial u}{\partial z}\right)^{2} \mathrm{~d} x .
$$

It is provided with a trace operator, linear and continuous for the natural topologies

$$
\gamma: W \rightarrow L^{2}\left(\Sigma_{1}\right) \times L^{2}\left(\Sigma_{2}\right), u \mapsto\left(u_{\mid \Sigma_{1}}, u_{\mid \Sigma_{2}}\right),
$$

so that the following notation is coherent: $W^{g}=\left\{u \in W, u_{\mid \Sigma_{1}}=g\right\}$.

Furthermore, for any Lipschitzian function $f$ from $\mathbb{R}$ into $\mathbb{R}$, the following chain rule holds for the weak derivatives: $\forall u \in W, f(u) \in W$ and

$$
\frac{\partial f(u)}{\partial z}=f^{\prime}(u) \frac{\partial u}{\partial z} \quad \text { a.e. in } \Omega,
$$

where $f^{\prime}$ indicates a bounded Borelian representative of $f$ derivative (it exists in the classic sense almost everywhere according to Rademacher's theorem). 


\subsection{Hypothesis}

One assumes that: $\exists c, C, M>0, \quad 0<c<C<1$,

$$
\begin{gathered}
c \leq \phi_{i} \leq C, \quad 0 \leq h \leq M \\
\forall \sigma_{1}, \sigma_{2} \in \mathbb{R}, \quad \sigma_{1}<\sigma_{2} \Rightarrow 0 \leq \phi\left(., ., \sigma_{2}\right)-\phi\left(., ., \sigma_{1}\right) \leq M\left(\sigma_{2}-\sigma_{1}\right) \\
\forall \sigma_{1}, \sigma_{2}, p_{1}, p_{2} \in \mathbb{R}, \quad\left|h_{i}\left(\sigma_{1}, p_{1}\right)-h_{i}\left(\sigma_{2}, p_{2}\right)\right| \leq M\left(\left|\sigma_{2}-\sigma_{1}\right|+\left|p_{2}-p_{1}\right|\right), \quad i=1,2,3 . \\
\sigma_{0} \in W^{0}, \quad \exists q>2, V^{P_{\Sigma_{1}}} \cap W^{1, q}(\Omega) \neq \emptyset, \quad g_{s} \in L^{\infty}\left(\Sigma_{1}\right), W^{g_{s}} \neq \emptyset .
\end{gathered}
$$

\subsection{Definition of a solution}

Definition 3.1. One calls solution to System $(13,16,15)$ for Conditions $(9)-(11)$ and $(12)$, any $\left(\sigma, v_{s}, p_{w}\right)$ in $W^{0} \times W^{g_{s}} \times V^{P_{\Sigma_{1}}}$ such that

(13) and (16) are satisfied a.e. in $\Omega$,

and, for any $\varphi$ in $V^{0}$,

$$
\int_{\Omega}{ }^{t} \overrightarrow{\nabla \varphi} K\left(., ., \sigma, p_{w}\right)\left(\overrightarrow{\nabla p}_{w}-\vec{B}\right) \mathrm{d} x+\int_{\Omega} \frac{\partial v_{s}}{\partial z} \varphi \mathrm{d} x=\int_{\Sigma_{2}} \phi(\sigma) v_{s} n_{z} \varphi \mathrm{d} \sigma .
$$

One has to remark that (12) is implicitly contained in the fact that the solutions belong to $W^{0}, W^{g_{s}}$ and $V^{P_{\Sigma_{1}}}$. Indeed, these functions possess the property of traces countinuity through interfaces $f_{i}$. It is also contained in the formulation (23) for the property of flux countinuity.

\subsection{Existence of a solution}

Proposition 3.2. There exists at least one solution to the problem in the sense of Definition 3.1.

In order to establish the existence of a solution, we suggest to use Schauder-Tikhonov's fixed point theorem, in the context of hilbertian separable spaces [10]. Therefore, one fixes $p_{w}$ in $V^{P_{\Sigma_{1}}}$. Thus, integration of equations (13) and (16) gives a solution $\sigma\left(p_{w}\right)$ in $W^{0}$ and a solution $v_{s}\left(p_{w}\right)$ in $W^{g_{s}}$. It is enough then to inject these two solutions in the following paralinearised version of equation (23)

$$
\int_{\Omega}{ }^{t} \overrightarrow{\nabla \varphi} K\left(., ., \sigma\left(p_{w}\right), p_{w}\right)\left(\vec{\nabla}_{w}-\vec{B}\right) \mathrm{d} x+\int_{\Omega} \frac{\partial v_{s}\left(p_{w}\right)}{\partial z} \varphi \mathrm{d} x=\int_{\Sigma_{2}} \phi(., ., \sigma) v_{s}\left(p_{w}\right) n_{z} \varphi \mathrm{d} \sigma,
$$

to obtain a unique solution $\overline{p_{w}}$ in $V^{P_{\Sigma_{1}}}$ and so, to build an application $\mathcal{S}$ from $V^{P_{\Sigma_{1}}}$ into himself, defined for any $p_{w}$ by $\mathcal{S}\left(p_{w}\right)=\overline{p_{w}}$.

It is immediate to notice that the solutions of System $(13,16,15)$, for the boundary conditions (9)-(11) and the interface condition (12), are the fixed points of $\mathcal{S}$.

One shows then that $\mathcal{S}$ keeps a non empty bounded closed convex set of $H^{1}(\Omega)$. It relies mainly on the fact that $v_{s}$ is bounded in $L^{\infty}(\Omega)$. From then on, with the help of a priori estimations, of subsequences extraction and of weak compactness arguments, one shows that $\mathcal{S}$ is weak-sequentially continuous. So, there exists a fixed point. The reader interested in technical details of these results is referred to [11].

\section{5. $L^{p}$ regularity of the obtained solution: an extension of N.G. Meyers's principle}

For many coupled non linear systems, the study of solution uniqueness passes by the treatment of trilinear term integration. From then on, it is necessary to improve the regularity knowledge of the obtained solution. This wellknown result, relaying on a disturbance of the Poisson equation, was initially introduces by [18]. Used again by $[4,19]$ and recently by [20] and [9] within the framework of diphasic models. We propose in [11] an adapted version to our problem, namely, an elliptic equation in a Lipschitzian domain, and of which a corollary is 
Proposition 3.3. There exists $p_{0}>2, k_{0}>0$, independent of solutions $\sigma$ and $v_{s}$ so that: $p_{w} \in W^{1, p_{0}}(\Omega)$ and $\left\|p_{w}\right\|_{W^{1, p_{0}}} \leq k_{0}$.

\subsection{Uniqueness of the solution}

\subsubsection{Presentation of the duality method}

Suggested by S.N. Antontsev's works [1] concerning the analytical study of diphasic filtration systems. The proposed method of uniqueness is based on a technique of transposition. It is inspired also by Holmgren's duality method, implemented by Oleŭnik [21].

This method of duality returns classically the study of the uniqueness of the primal problem to the study of the existence of a solution to the dual problem, in compatible functional frameworks.

We denote $\left(\sigma_{1}, p_{1}, v_{1}\right)$ and $\left(\sigma_{2}, p_{2}, v_{2}\right)$ two solutions to system $(13,16,15)$ with the boundary conditions $(9)-$ (11) and (12).

After subtracting of equations $(13,16)$ and $(23)$ verified by $\left(\sigma_{1}, p_{1}, v_{1}\right)$ and $\left(\sigma_{2}, p_{2}, v_{2}\right)$, we shall transport the derivations on the test functions, in order to introduce quantities $\bar{\sigma}=\sigma_{1}-\sigma_{2}, \bar{v}=v_{1}-v_{2}$ and $\bar{p}=p_{1}-p_{2}$. In order to relieve the demonstration writing, we introduce some notations

$$
\begin{aligned}
& \phi^{\prime}=\frac{\phi\left(., ., \sigma_{1}\right)-\phi\left(., ., \sigma_{2}\right)}{\sigma_{1}-\sigma_{2}} \quad \text { if } \sigma_{1} \neq \sigma_{2}, \quad \phi^{\prime}=\frac{\partial \phi}{\partial \sigma}\left(., ., \sigma_{1}\right) \\
& F^{\prime}=\frac{F\left(., ., \sigma_{1}\right)-F\left(., ., \sigma_{2}\right)}{\sigma_{1}-\sigma_{2}} \quad \text { if } \sigma_{1} \neq \sigma_{2}, \quad F^{\prime}=\frac{\partial F}{\partial \sigma}\left(., ., \sigma_{1}\right) \\
& D_{1} K=\frac{K\left(x, z, \sigma_{2}, p_{1}\right)-K\left(x, z, \sigma_{2}, p_{2}\right)}{\bar{p}} \quad \text { if } \overline{\mathrm{p}} \neq 0, \quad D_{1} K=\frac{\partial K\left(x, z, \sigma_{2}, p_{2}\right)}{\partial p} \quad \text { if } \overline{\mathrm{p}}=0 \text {, } \\
& D_{2} K=\frac{K\left(x, z, \sigma_{1}, p_{1}\right)-K\left(x, z, \sigma_{2}, p_{1}\right)}{\bar{\sigma}} \quad \text { if } \bar{\sigma} \neq 0, \quad \quad D_{2} K=\frac{\partial K\left(x, z, \sigma_{1}, p_{1}\right)}{\longrightarrow \partial \sigma \rightarrow} \quad \text { if } \bar{\sigma}=0 \text {, }
\end{aligned}
$$

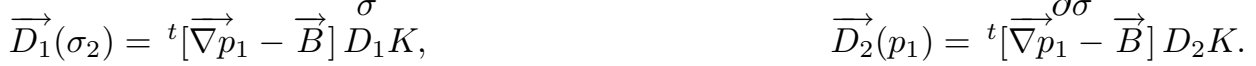

Since functions $\phi_{i}, F_{i}, k_{i}$ and $h_{i}$ are Lipschitzian, $\phi^{\prime}$ and $F^{\prime}$ are measurable functions. According to Rade macher's theorem, one is even able to choose them as bounded Borelian functions. Also, $\overrightarrow{D_{2}}\left(p_{1}\right)$ and $\overrightarrow{D_{1}}\left(\sigma_{2}\right)$ are $\left[L^{2}(\Omega)\right]^{2}$ functions. More exactly, thanks to Proposition 3.3, they are elements of $\left[L^{p_{0}}(\Omega)\right]^{2}$.

Right now, it is necessary for us to clarify the functional spaces required to look for the solutions to the dual problem and to legitimize Gauss-Green formulae. For it, one considers

$$
X=\left\{u \in L^{2}(\Omega), \forall i, \frac{\partial u}{\partial z} \in L^{2}\left(\Omega_{i}\right)\right\}, \quad\|u\|_{X}^{2}=\|u\|_{L^{2}(\Omega)}^{2}+\sum_{i}|| \frac{\mathrm{d} u}{\mathrm{~d} z}||_{L^{2}\left(\Omega_{i}\right)}^{2} .
$$

It is a subspace of

$$
\overline{B V_{z}}=\left\{u \in L^{2}(\Omega), \frac{\partial u}{\partial z} \in \mathcal{M}_{b}(\Omega)\right\}
$$

(where $\mathcal{M}_{b}(\Omega)$ indicates the set of bounded Radon measures on $\Omega$ ). It has been introduced by [28] for the study of degenerated hyperbolic-parabolic problems. We suggest to denote by $\frac{\mathrm{d} u}{\mathrm{~d} z}$ the absolutely continuous part, with respect to the Lebesgue measure $\mathcal{L}$, of the measure $\frac{\partial u}{\partial z}$. So, for any $u$ of $X$,

$$
\frac{\mathrm{d} u}{\mathrm{~d} z}=\sum_{i} \frac{\partial u_{\mid \Omega_{i}}}{\partial z} \mathbb{I}_{\Omega_{i}}
$$

while

$$
\frac{\partial u}{\partial z}=\frac{\mathrm{d} u}{\mathrm{~d} z} \mathrm{~d} \mathcal{L}+\sum_{i}\left(u^{+}-u^{-}\right) n_{z} \mathcal{H}^{1}\left\llcorner f_{i}\right.
$$


where $u^{+}$and $u^{-}$represent the traces of $u$ on the right and on the left of $f_{i}$ (if one chooses the direction given by $\vec{n}$, the normal vector to $f_{i}$, from $\Omega_{i}$ to $\Omega_{i+1}$ ) and $\mathcal{H}^{1}\left\llcorner f_{i}\right.$ represents the restriction of the 1-dimensionnal Hausdorff measure on $f_{i}$.

$$
Y_{p}=\left\{v \in L^{2}(\Omega), \forall i, \frac{\partial v}{\partial z} \in L^{q}\left(\Omega_{i}\right)\right\}, \quad q=\frac{2 p}{p+2} .
$$

\subsubsection{First step: superimposing of the balance states and transposition}

Concerning equations verified by $v_{1}$ and $v_{2}$ : the subtraction of the equations, the integration by parts on each $\Omega_{i}$, by adding of the $i$ together and by regrouping each terms, it comes, for any $\alpha$ of $X$,

$$
\begin{aligned}
\int_{\Omega} \bar{\sigma} \phi^{\prime}\left(v_{2} \frac{\mathrm{d} \alpha}{\mathrm{d} z}-\frac{\alpha}{h}\right) \mathrm{d} x+\int_{\Omega} \bar{v}\left(\phi\left(., ., \sigma_{1}\right)-1\right) \frac{\mathrm{d} \alpha}{\mathrm{d} z} \mathrm{~d} x & +\sum_{i} \int_{f_{i}} \bar{\sigma} v_{2}\left[\phi^{\prime+} \alpha^{+}-\phi^{\prime-} \alpha^{-}\right] n_{z} \mathrm{~d} \sigma \\
-\sum_{i} \int_{f_{i}} \bar{v}\left(1-\phi^{+}\left(., ., \sigma_{1}\right)\right) \alpha^{+} n_{z} \mathrm{~d} \sigma & +\sum_{i} \int_{f_{i}} \bar{v}\left(1-\phi^{-}\left(., ., \sigma_{1}\right)\right) \alpha^{-} n_{z} \mathrm{~d} \sigma \\
& =\int_{\Sigma_{2}} \bar{\sigma} \phi^{\prime} v_{2} \alpha n_{z} \mathrm{~d} \sigma-\int_{\Sigma_{2}} \bar{v}\left(1-\phi\left(., ., \sigma_{1}\right)\right) \alpha n_{z} \mathrm{~d} \sigma .
\end{aligned}
$$

Concerning equations verified by $\sigma_{1}$ and $\sigma_{2}$ : in a same way, one has, for any $\beta$ of $Y_{p_{0}}$ ( $p_{0}$ given by Property 3.3),

$$
\int_{\Omega} \bar{\sigma}\left(\frac{\mathrm{d} \beta}{\mathrm{d} z}+F^{\prime} \beta\right) \mathrm{d} x+\int_{\Omega} \bar{p} \frac{\mathrm{d} \beta}{\mathrm{d} z} \mathrm{~d} x=\sum_{i} \int_{f_{i}}(\bar{p}+\bar{\sigma})\left(\beta^{+}-\beta^{-}\right) \eta_{z} \mathrm{~d} \sigma+\int_{\Sigma_{2}}(\bar{p}+\bar{\sigma}) \beta \eta_{z} \mathrm{~d} \sigma .
$$

Concerning the variational equations verified by $p_{1}$ and $p_{2}$, a subtraction gives for any $\gamma$ of $V^{0}$,

$$
\begin{aligned}
\int_{\Omega} \bar{\sigma} \vec{D}_{2}\left(p_{1}\right) \overrightarrow{\nabla \gamma}+\bar{p} \vec{D}_{1}\left(\sigma_{2}\right) \overrightarrow{\nabla \gamma} \mathrm{d} x+\int_{\Omega} t \overrightarrow{\nabla \gamma} K\left(., ., \sigma_{2}, p_{2}\right) \overrightarrow{\nabla \bar{p}} \mathrm{~d} x+\int_{\Sigma_{2}} \bar{v} \gamma n_{z} \mathrm{~d} \sigma= \\
\int_{\Omega} \bar{v} \frac{\partial \gamma}{\partial z} \mathrm{~d} x+\int_{\Sigma_{2}} \bar{\sigma} \phi^{\prime} v_{2} n_{z} \gamma \mathrm{d} \sigma+\int_{\Sigma_{2}} \bar{v} \phi\left(., ., \sigma_{1}\right) n_{z} \gamma \mathrm{d} \sigma .
\end{aligned}
$$

From then on, regrouping equations $(25,26)$ and $(27)$ allows us to obtain the vectorial variational formulation on $X \times Y_{p_{0}} \times V^{0}$,

$$
\begin{aligned}
& \int_{\Omega} \bar{\sigma}\left\{\phi^{\prime}\left(v_{2} \frac{\mathrm{d} \alpha}{\mathrm{d} z}-\frac{\alpha}{h}\right)+\frac{\mathrm{d} \beta}{\mathrm{d} z}\right\} \mathrm{d} x+\int_{\Omega} \bar{\sigma}\left\{F^{\prime} \beta+\vec{D}_{2}\left(p_{1}\right) \overrightarrow{\nabla \gamma}\right\} \mathrm{d} x-\int_{\Omega} \bar{v}\left\{\left(1-\phi\left(., ., \sigma_{1}\right)\right) \frac{\mathrm{d} \alpha}{\mathrm{d} z}+\frac{\partial \gamma}{\partial z}\right\} \mathrm{d} x \\
& +\int_{\Omega} \bar{p}\left\{\frac{\mathrm{d} \beta}{\mathrm{d} z}+\vec{D}_{1}\left(\sigma_{2}\right) \overrightarrow{\nabla \gamma}\right\} \mathrm{d} x+\int_{\Omega}{ }^{t} \overrightarrow{\nabla \gamma} K\left(., ., \sigma_{2}, p_{2}\right) \overrightarrow{\nabla \bar{p}} \mathrm{~d} x \\
& +\sum_{i} \int_{f_{i}} \bar{\sigma} v_{2}\left[\phi^{\prime+} \alpha^{+}-\phi^{\prime-} \alpha^{-}\right] n_{z} \mathrm{~d} \sigma-\sum_{i} \int_{f_{i}} \bar{\sigma}\left[\beta^{+}-\beta^{-}\right] n_{z} \mathrm{~d} \sigma \\
& -\sum_{i} \int_{f_{i}} \bar{v}\left[1-\phi^{+}\left(., ., \sigma_{1}\right)\right] \alpha^{+} n_{z} \mathrm{~d} \sigma+\sum_{i} \int_{f_{i}} \bar{v}\left[1-\phi^{-}\left(., ., \sigma_{1}\right)\right] \alpha^{-} n_{z} \mathrm{~d} \sigma-\sum_{i} \int_{f_{i}} \bar{p}\left\{\beta^{+}-\beta^{-}\right\} n_{z} \mathrm{~d} \sigma \\
& =\int_{\Sigma_{2}} \bar{\sigma}\left\{\beta+\phi^{\prime} v_{2}(\alpha+\gamma)\right\} n_{z} \mathrm{~d} \sigma+\int_{\Sigma_{2}} \bar{v}\left\{\left(1-\phi\left(., ., \sigma_{1}\right)\right)(\alpha+\gamma)\right\} \eta_{z} \mathrm{~d} \sigma+\int_{\Sigma_{2}} \bar{p} \beta \eta_{z} \mathrm{~d} \sigma .
\end{aligned}
$$


Thus, it is enough to find $(\alpha, \beta, \gamma)$ in $X \times Y_{P_{0}} \times V^{0}$, solution to the formal dual (or transposed) problem in $\Omega$

$$
\begin{aligned}
& \left(1-\phi\left(., ., \sigma_{1}\right)\right) \frac{\mathrm{d} \alpha}{\mathrm{d} z}+\frac{\partial \gamma}{\partial z}=0, \\
& \phi^{\prime}\left(v_{2} \frac{\mathrm{d} \alpha}{\mathrm{d} z}-\frac{\alpha}{h}\right)+\left(\frac{\mathrm{d} \beta}{\mathrm{d} z}+F^{\prime} \beta\right)+\vec{D}_{2}\left(p_{1}\right) \overrightarrow{\nabla \gamma}=0, \\
& \frac{\partial \beta}{\partial z}+\vec{D}_{1}\left(\sigma_{2}\right) \overrightarrow{\nabla \gamma}-\operatorname{Div}\left\{K\left(., ., \sigma_{2}, p_{2}\right) \overrightarrow{\nabla \gamma}\right\}=\bar{p},
\end{aligned}
$$

with the interface condition on $f_{i}$

$$
\left[1-\phi^{+}\left(., ., \sigma_{1}\right)\right] \alpha^{+}=\left[1-\phi^{-}\left(., ., \sigma_{1}\right)\right] \alpha^{-}, \quad \beta^{+}-\beta^{-}=v_{2}\left[\phi^{\prime+} \alpha^{+}-\phi^{--} \alpha^{-}\right]
$$

and the boundary conditions

$$
\gamma=0 \text { on } \Sigma_{1}, \quad \beta=0, \alpha=-\gamma \text { on } \Sigma_{2}, \quad-{ }^{\mathrm{t}} \vec{n} \mathrm{~K}\left(\sigma_{2}, \mathrm{p}_{2}\right) \overrightarrow{\nabla \gamma}=0 \text { on } \partial \Omega \backslash \Sigma_{1}
$$

The dual system (29)-(31) is linear, but it is strongly coupled (by the equations, as well as by the boundary conditions).

\subsubsection{Second step: existence of a solution to the dual problem}

We are going to consider here a method by substitution. This method allows us to reduce the dual system to a linear equation concerning $\gamma$, the dual unknown of the pressure, in the framework of the classical LaxMilgram's theorem. This theorem, which gives a sufficient condition of existence and uniqueness of a solution, can be too constraining. One can prefer Brezzi-Babuška's theorem ([7], p. 564 for example). But in our case, because of the coupling, the inf sup conditions do not seem immediate to obtain.

Let $\gamma$ be fixed in $V^{0}$. By a simple quadrature of a linear differential equation on every $\Omega_{i}$ (and by using conditions on $\Sigma_{2}$ and on $f_{i}$ ), one obtains the existence and the uniqueness of the solution $\alpha[\gamma]$ in $X$ to equation (29) with conditions (32) and (33).

Furthermore, the application $\alpha: V^{0} \rightarrow X, \gamma \mapsto \alpha[\gamma]$ is linear and continuous [11].

The same work allows us to obtain a result of existence and uniqueness of $\beta[\gamma]$ in $Y_{p_{0}}$ solution to

$$
\begin{aligned}
& \frac{\mathrm{d} \beta}{\mathrm{d} z}[\gamma]+F^{\prime} \beta[\gamma]+H[\gamma]=0 \text { in } \Omega \text { where } H[\gamma]=\phi^{\prime}\left(v_{2} \frac{\mathrm{d} \alpha}{\mathrm{d} z}[\gamma]-\frac{\alpha[\gamma]}{h}\right)+D_{2}\left(p_{1}\right) \nabla \gamma, \\
& \beta^{+}-\beta^{-}=v_{2}\left[\phi^{\prime+} \alpha^{+}-\phi^{\prime-} \alpha^{-}\right] \text {on } \mathrm{f}_{\mathrm{i}}, \quad \beta[\gamma]=0 \text { on } \Sigma_{2} \text {. }
\end{aligned}
$$

One obtains this solution in $Y_{p_{0}}$ since, $\nabla p_{1} \in L^{p}(\Omega), \nabla \gamma \in\left[L^{p_{0}}(\Omega)\right]^{2}$ and $\vec{D}_{2}\left(p_{1}\right) \overrightarrow{\nabla \gamma} \in L^{q_{0}}(\Omega)$ where $q_{0}=\frac{2 p_{0}}{p_{0}+2}$.

Also, the application $\beta: V^{0} \rightarrow Y_{p_{0}}, \gamma \mapsto \beta[\gamma]$ is linear and continuous [11].

We are henceforth able to give the dual variational problem and to look for a solution by means of LaxMilgram's theorem. In order to do so, and in agreement with the modelling, we suppose that $\left\|\phi^{\prime}\right\|_{\infty},\left\|D_{2} K\right\|_{\infty}$ 
and $\left\|D_{1} K\right\|_{\infty}$ are small, in a sense precised in the sequel. We note now

$$
(u, v) \mapsto \int_{\Omega} t \overrightarrow{\nabla v} K\left(\sigma_{2}, p_{2}\right) \overrightarrow{\nabla u} \mathrm{~d} x+\int_{\Omega} v \vec{D}_{1}\left(\sigma_{2}\right) \overrightarrow{\nabla u} \mathrm{~d} x+\int_{\Omega} v \frac{\mathrm{d} \beta}{\mathrm{d} z}[u] \mathrm{d} x+\sum_{i} \int_{f_{i}}\left(\beta^{+}[u]-\beta^{-}[u]\right) v n_{z} \mathrm{~d} \sigma .
$$

As $\beta[u]$ and $\sigma[u]$ are linear with respect to $u, b$ is a bilinear form.

Furthermore, as in dimension 2, the injection of $H^{1}(\Omega)$ in $L^{q^{\prime}}(\Omega)$ is compact $\left(\frac{1}{q}+\frac{1}{q^{\prime}}=1\right), b$ is continuous on $V^{0} \times V^{0}$, for its natural topology. Thus, for any $u$ in $V^{0}$, if $a=n_{w} \inf \left(\lambda_{a}, \lambda_{s}\right)$, one has

$$
b(u, u) \geq a\|\overrightarrow{\nabla u}\|^{2}-c\left(\left\|\phi^{\prime}\right\|_{\infty}+\left\|D_{2} K\right\|_{\infty}+\left\|D_{1} K\right\|_{\infty}\right)\|u\|_{V^{0}}^{2}
$$

and as Poincaré's inequality is valid in $V^{0}$, one gets,

$$
\forall u \in V^{0}, \quad b(u, u) \geq a\|u\|_{V^{0}}^{2}-c\left(\left\|\phi^{\prime}\right\|_{\infty}+\left\|D_{2} K\right\|_{\infty}+\left\|D_{1} K\right\|_{\infty}\right)\|u\|_{V^{0}}^{2} .
$$

From then on, hypothesis concerning the smallness of $\left\|\phi^{\prime}\right\|_{\infty},\left\|D_{2} K\right\|_{\infty}$ and $\left\|D_{1} K\right\|_{\infty}$, which finds here its implicit formulation, insures the coercitivity of the form $b$ and so, the existence of a solution to the dual system.

In conclusion, there exists $(\alpha, \beta, \gamma)$ in $X \times Y_{p_{0}} \times V^{0}$, verifying the equations (29) and (30) with the conditions (32) and (33), and for any $\varphi$ of $V^{0}$, the variational equation,

$$
\int_{\Omega} \varphi\left[\frac{\mathrm{d} \beta}{\mathrm{d} z}[\gamma]+\vec{D}_{1}\left(\sigma_{2}\right) \overrightarrow{\nabla \gamma}\right] \mathrm{d} x+\int_{\Omega}{ }^{t} \overrightarrow{\nabla \gamma} K\left(\sigma_{2}, p_{2}\right) \overrightarrow{\nabla \varphi} \mathrm{d} x+\sum_{i} \int_{f_{i}} v_{2}\left(\phi^{\prime+} \alpha^{+}[u]-\phi^{\prime-} \alpha^{-}[u]\right) v n_{z} \mathrm{~d} \sigma=\langle\bar{p}, \varphi\rangle .
$$

In particular, $\varphi=\bar{p}$ in (28) leads to $\|\bar{p}\|_{L^{2}(\Omega)}^{2}=0$ and to $p_{1}=p_{2}$ a.e.

As $\sigma$ and $v_{s}$ are univocaly obtained for a fixed $p_{w}$, one has shown the uniqueness of the solution $\left(\sigma, v_{s}, p_{w}\right)$.

\subsubsection{Alternatives}

Another approach consists in applying Fredholm's alternative as presented in [6] and [12]. This theorem, concerning the wellknown compatibility conditions of linear systems in finite dimension, becomes widespread in the case of compact operators. We are going to develop this method of uniqueness in the next section. A third one is the method of artificial compressibility where one suggests to replace the elliptic equation

$$
\frac{\partial \beta}{\partial z}+\vec{D}_{1}\left(\sigma_{2}\right) \overrightarrow{\nabla \gamma}-\operatorname{Div}\left\{K\left(\sigma_{2}, p_{2}\right) \overrightarrow{\nabla \gamma}\right\}=\bar{p}
$$

by the parabolic regularization (effect of very weak compressibility)

$$
\varepsilon \frac{\partial \gamma}{\partial t}+\frac{\partial \beta}{\partial z}+\vec{D}_{1}\left(\sigma_{2}\right) \overrightarrow{\nabla \gamma}-\operatorname{Div}\left\{K\left(\sigma_{2}, p_{2}\right) \overrightarrow{\nabla \gamma}\right\}=\bar{p} .
$$

On the one hand, one needs less hypotheses on the operator (for the existence of a solution) in the parabolic case than in the elliptic case. On the other hand, one needs a priori estimations, on the obtained solution $\gamma_{\varepsilon}$, and needs to pass to the limit on $\varepsilon$, as $\varepsilon \rightarrow 0^{+}$.

Finally, a last approach consists in using for this system, although linear, a fixed point method, as the one proposed in the section: existence of a solution. 
It consists, for any fixed $\gamma$ in $V^{0}$, in resolving in $V^{0}$

$$
\frac{\partial \beta}{\partial z}[\gamma]+\vec{D}_{1}\left(\sigma_{2}\right) \overrightarrow{\nabla \theta}-\operatorname{Div}\left\{K\left(\sigma_{2}, p_{2}\right) \overrightarrow{\nabla \theta}\right\}=\bar{p}
$$

and in studying the properties of Application $\mathcal{S}: \gamma \mapsto \theta$.

As the equations are linear, it is the same for $\mathcal{S}$. As the previous study proves that $\mathcal{S}$ is continuous, it is weakly sequentially continuous from $V^{0}$ into $V^{0}$. So one has to find a non empty bounded closed convex set, invariant by $\mathcal{S}$.

By summing up the study of the second step, it comes at once

$$
\|\theta\|_{V^{0}} \leq c|| \bar{p}\left\|+c\left(\left\|\phi^{\prime}\right\|_{\infty}+\left\|D_{2} K\right\|_{\infty}+\left\|D_{1} K\right\|_{\infty}\right)\right\| \gamma \|_{V^{0}},
$$

so that if $\eta=c\left(\left\|\phi^{\prime}\right\|_{\infty}+\left\|D_{2} K\right\|_{\infty}+\left\|D_{1} K\right\|_{\infty}\right)<1$, the looked for convex is $V^{0} \cap \bar{B}\left(0, \frac{c\|\bar{p}\|}{1-\eta}\right)$.

Even there, hypothesis concerning the smallness of certain coefficients in needed to guarantee that the invariance of a convex set is gathered.

\section{A SECOND CASE: General AMPlitude COMPACTION}

In this second approach of the discretisation, one does not need any more the smallness of certain coefficients. On the other hand, one considers that $\phi_{i}$ is a regular function of arguments $x, z$ and $\sigma$ and that the function $\phi: \phi(x, z, \sigma)=\sum_{i} \phi_{i}(x, z, \sigma) \mathbb{I}_{\Omega_{i}}(x, z)$, is continuous.

We propose, for this problem, an approach of predictive-corrective type. That is, if one still denotes by $h$ the iterative step and $\sigma_{0}$ the datum of $\sigma$ at the previous iteration, and if one supposes $h$ large enough ${ }^{1}$ to neglect $\frac{\partial \phi}{\partial t}$ (asymptotically stable system), one looks for $\left(\sigma, v_{s}, p_{w}\right)$ in $W^{0} \times W^{g_{s}} \times V^{P_{\Sigma_{1}}}$ solution to

$$
\begin{gathered}
\frac{\partial \sigma}{\partial z}=F\left(., ., \sigma_{0}\right)-\frac{\partial p_{w}}{\partial z} \\
\frac{\partial(1-\phi(., ., \sigma)) v_{s}}{\partial z}=0 \text { on } \Omega_{i} \\
-\operatorname{div}\left\{K\left(., ., \sigma, p_{w}\right)\left(\overrightarrow{\nabla p_{w}}-\vec{B}\right)\right\}+\frac{\partial}{\partial z} v_{s}=0
\end{gathered}
$$

with the same boundary and interfaces conditions.

After an integration of the two first equations, one calls solution to the system any $\left(\sigma, v_{s}, p_{w}\right)$ in $W^{0} \times W^{g_{s}} \times$ $V^{P_{\Sigma_{1}}}$ such that:

$$
\begin{gathered}
\sigma(x, z)=\int_{\Sigma_{1}(x)}^{z} F\left(., ., \sigma_{0}(x, s)\right) d s-p_{w}(x, z)+P_{\Sigma_{1}}\left(x, \Sigma_{1}(x)\right), \\
v_{s}(x, z)=\frac{1-\phi\left(x, \Sigma_{1}(x), 0\right)}{1-\phi(x, z, \sigma(x, z))} g_{s}\left(x, \Sigma_{1}(x)\right), \\
\forall \varphi \in V^{0}, \quad \int_{\Omega}{ }^{t} \overrightarrow{\nabla \varphi} K\left(., ., \sigma, p_{w}\right)\left(\overrightarrow{\nabla p}_{w}-\vec{B}\right) \mathrm{d} x+\int_{\Omega} \frac{\partial v_{s}}{\partial z} \varphi \mathrm{d} x=\int_{\Sigma_{2}} \phi(., ., \sigma) v_{s} n_{z} \varphi \mathrm{d} \sigma .
\end{gathered}
$$

As for the first case, a fixed point method leads directly to the existence of a solution.

\footnotetext{
${ }^{1}$ One works with geologic scale and $h$ may represent a century.
} 


\subsection{Uniqueness of the solution}

The method used in this section is closed to the one already proposed in Section 3.6.

Using the same calculus as in the first step of this duality method, one is led to look for $(\alpha, \beta, \gamma)$ a priori in $X \times Y_{p_{0}} \times V^{0}$ solution to System

$$
\begin{gathered}
\left(1-\phi\left(., ., \sigma_{1}\right)\right) \frac{\mathrm{d} \alpha}{\mathrm{d} z}+\frac{\partial \gamma}{\partial z}=0, \\
\frac{\mathrm{d} \beta}{\mathrm{d} z}+\phi^{\prime} v_{2} \frac{\mathrm{d} \alpha}{\mathrm{d} z}+\overrightarrow{D_{2}}\left(p_{1}\right) \overrightarrow{\nabla \gamma}=0, \\
\frac{\partial \beta}{\partial z}-\operatorname{Div}\left(K\left(., ., \sigma_{2}, p_{2}\right) \overrightarrow{\nabla \gamma}\right)+\overrightarrow{D_{1}}\left(\sigma_{2}\right) \overrightarrow{\nabla \gamma}=\bar{p},
\end{gathered}
$$

with the interface conditions on $f_{i}$

$$
\left[1-\phi^{+}\left(., ., \sigma_{1}\right)\right] \alpha^{+}=\left[1-\phi^{-}\left(., ., \sigma_{1}\right)\right] \alpha^{-}, \quad \beta^{+}-\beta^{-}=v_{2}\left[\phi^{\prime+} \alpha^{+}-\phi^{\prime-} \alpha^{-}\right],
$$

and the boundary conditions $([3])$

$$
\begin{aligned}
\gamma=0 & \text { on } \Sigma_{1}, \\
\beta=0, \quad \alpha=-\gamma & \text { on } \Sigma_{2}, \\
-{ }^{t} \vec{n} K\left(., ., \sigma_{2}, p_{2}\right) \nabla \gamma=0 & \text { on } \partial \Omega \backslash \Sigma_{1} .
\end{aligned}
$$

But as $\phi^{+}=\phi^{-}$and $\phi^{+}=\phi^{-}$( $\phi$ is supposed continuous), the interface conditions on $f_{i}$ become $\alpha^{+}=\alpha^{-}$ and $\beta^{+}=\beta^{-}$.

From then on, $\alpha$ and $\beta$ are looked for in $W$ and an algebraic manipulation leads us to look for $\gamma$ in $V^{0}$ solution to

$$
-\operatorname{Div}\left(K\left(., ., \sigma_{2}, p_{2}\right) \overrightarrow{\nabla \gamma}\right)+\vec{D}_{1}\left(\sigma_{2}\right) \overrightarrow{\nabla \gamma}+\frac{\phi^{\prime} v_{2}}{1-\phi\left(., ., \sigma_{1}\right)} \frac{\partial \gamma}{\partial z}-\vec{D}_{2}\left(p_{1}\right) \overrightarrow{\nabla \gamma}=\bar{p}
$$

i.e.

$$
-\operatorname{Div}(A \overrightarrow{\nabla \gamma})+\overrightarrow{\mathfrak{B}} \cdot \overrightarrow{\nabla \gamma}=\bar{p}
$$

with the boundary conditions of edge

$$
\gamma=0 \text { on } \Sigma_{1}, \quad-{ }^{t} \vec{n} K\left(., ., \sigma_{2}, p_{2}\right) \overrightarrow{\nabla \gamma}=0 \text { on } \partial \Omega \backslash \Sigma_{1} .
$$

Then, the second step is based on an application of Fredholm's alternative.

To conclude, we consider the following family of bilinear forms $\left(a_{\lambda}\right)_{\lambda \geq 0}$ on $V \times V$

$$
a_{\lambda}: V \times V \rightarrow \mathbb{R}, \quad(u, v) \mapsto \int_{\Omega} t \overrightarrow{\nabla u} A \overrightarrow{\nabla v} \mathrm{~d} x+\int_{\Omega} \overrightarrow{\mathfrak{B}} \cdot \overrightarrow{\nabla u} v \mathrm{~d} x+\lambda \int_{\Omega} u v \mathrm{~d} x,
$$

and $a$ instead of $a_{0}$. We notice that as $\overrightarrow{\mathfrak{B}} \in\left(L^{p_{0}}(\Omega)\right)^{2}$, for any $u$ in $V, \overrightarrow{\mathfrak{B}} \cdot \overrightarrow{\nabla u} \in L^{q_{0}}(\Omega)$. So thanks to the continuous injection of $V$ in $L^{q_{0}^{\prime}}(\Omega)$, the trilinear term $\int_{\Omega} \overrightarrow{\mathfrak{B}} \cdot \overrightarrow{\nabla u} v \mathrm{~d} x$ has a sense for any $v$ of $V$ and $a_{\lambda}$ is continuous. Furthermore, one shows that there is a value of $\lambda$ such that $a_{\lambda}$ is $V^{0}$-elliptic. So, for any $f$ in $L^{2}(\Omega)$, there exists a unique $u$ in $V^{0}$ such that

$$
\forall v \in V, \quad a_{\lambda}(u, v)=\int_{\Omega} f v \mathrm{~d} x .
$$


From then on, a classic application of Fredholm's alternative [6] allows us to show that the problem: find $u$ in $V^{0}$ such that

$$
\forall v \in V, \quad a(u, v)=\int_{\Omega} f v \mathrm{~d} x,
$$

admits a solution, moreover unique, if the trivial solution is the only solution to (39) when $f=0$.

This last result ensues from the weak maximum principle [12], generalized in $\Omega \subset \mathbb{R}^{N}$, as follows:

In a first step, one shows that $u$ is upper bounded. We consider $k$ such that $0 \leq k<\gamma=\underset{\Omega}{\sup e s s} u$ (possibly infinite) and the test function $v_{k}=(u-k)^{+}$in (39). It follows the estimation,

$$
\left.c|| \overrightarrow{\nabla v}{ }_{k}\left\|_{L^{2}(\Omega)^{N}}^{2} \leq\right\| \overrightarrow{\mathfrak{B}} \|_{L^{p_{0}}(\Omega)}\right)\left\|\overrightarrow{\nabla v_{k}}\right\|_{L^{2}(\Omega)^{N}}\left\|v_{k}\right\|_{L^{q_{0}^{\prime}}(\Omega)},
$$

and, since by construction $v_{k}$ is not null, it comes, for a big enough value of $p^{*}$,

$$
\|\overrightarrow{\nabla v}\|_{L^{2}(\Omega)^{N}} \leq C\left\|v_{k}\right\|_{L^{q_{0}^{\prime}(\Omega)}} \leq C\left[\operatorname{meas}\left(\operatorname{supp} v_{k}\right)\right]^{\frac{p^{*}-q_{0}}{p^{*} q_{0}^{\prime}}}\left\|v_{k}\right\|_{L^{p^{*}}(\Omega)},
$$

where $C$ represents an arbitrary constant. Finally, the countinuous injection of $V^{0}$ in $L^{p^{*}}(\Omega)$ leads to

$$
\left\|\overrightarrow{\nabla v}_{k}\right\|_{L^{2}(\Omega)^{N}} \leq C\left[\operatorname{meas}\left(\operatorname{supp} v_{k}\right)\right]^{\frac{p^{*}-q_{0}}{p^{*} q_{0}^{0}}}\left\|\overrightarrow{\nabla v}_{k}\right\|_{L^{2}(\Omega)^{N}}
$$

So $\mathcal{L}^{N}-\operatorname{meas}\left(\operatorname{supp} v_{k}\right) \geq C>0$, constant independent of $k\left(\left\|\vec{\nabla} \vec{v}_{k}\right\|_{L^{2}(\Omega)^{N}}>0\right)$. By noticing that $\operatorname{supp} v_{k} \searrow$ $\operatorname{supp} v_{\gamma}$ when $k \nearrow \gamma$, it ensues from Beppo-Lévi's theorem that

$$
\operatorname{meas}\left(\operatorname{supp} v_{\gamma}\right)>0 \text {. }
$$

So, there is a non negligeable $\mathcal{L}^{N}$-measurable subset $\Omega_{0}$ of $\Omega$, such that $u=\gamma$ a.e. in $\Omega_{0}$. Moreover, since $u$ in $\mathcal{L}^{N}$-integrable on $\Omega, \gamma<+\infty$.

Second step consists in showing that $u$ is non positive $\mathcal{L}^{N}$-a.e. in $\Omega$. In order to do so, let us consider, for any $\varepsilon>0$, the test function $v_{\varepsilon}=\frac{u^{+}}{\gamma+\varepsilon-u^{+}}$. From then on, it comes

$$
\int_{\Omega} \frac{\gamma+\varepsilon}{\left(\gamma+\varepsilon-u^{+}\right)^{2}} t^{t} \overrightarrow{\nabla u^{+}} A \overrightarrow{\nabla u^{+}} \mathrm{d} x+\int_{\Omega} \overrightarrow{\mathfrak{B}} \cdot \overrightarrow{\nabla u^{+}} \frac{u^{+}}{\gamma+\varepsilon-u^{+}} \mathrm{d} x=0
$$

and

$$
c \int_{\Omega} \frac{\left|\overrightarrow{\nabla u^{+}}\right|^{2}}{\left(\gamma+\varepsilon-u^{+}\right)^{2}} \mathrm{~d} x \leq \int_{\Omega}|\overrightarrow{\mathfrak{B}}| \frac{\left|\overrightarrow{\nabla u^{+}}\right|}{\gamma+\varepsilon-u^{+}} \frac{u^{+}}{\gamma+\varepsilon} \mathrm{d} x .
$$

One observes that, $0 \leq \frac{u^{+}}{\gamma+\varepsilon} \leq 1$. So, if one notes $w_{\varepsilon}=\ln \left[\frac{\gamma+\varepsilon}{\gamma+\varepsilon-u^{+}}\right]$, then $w_{\varepsilon} \in V^{0}$, the chain rule gives $\overrightarrow{\nabla w}_{\varepsilon}=\frac{\overrightarrow{\nabla u^{+}}}{\gamma+\varepsilon-u^{+}}$and one gets

$$
c \int_{\Omega}\left|\overrightarrow{\nabla w}_{\varepsilon}\right|^{2} \mathrm{~d} x \leq \int_{\Omega}|\overrightarrow{\mathfrak{B}}|\left|\overrightarrow{\nabla w}_{\varepsilon}\right| \mathrm{d} x \leq|| \overrightarrow{\mathfrak{B}} \|\left._{L^{2}(\Omega)}|| \overrightarrow{\nabla w}_{\varepsilon}\right|_{L^{2}(\Omega)^{N}}
$$

In conclusion, by means of Poincaré's inequality, for any $\varepsilon>0,\left\|w_{\varepsilon}\right\|_{L^{2}(\Omega)} \leq c\left\|\overrightarrow{\nabla w_{\varepsilon}}\right\|_{L^{2}(\Omega)^{N}} \leq C$ (constant independent of $\varepsilon$ ). 
If one supposes, by contradiction, the negation of the assertion: $u \leq 0$ a.e., $\gamma>0$ and it ensues directly that

$$
\begin{aligned}
\left(\ln \left[\frac{\gamma+\varepsilon}{\varepsilon}\right]\right)^{2} \operatorname{meas}\left(\Omega_{0}\right) & =\int_{\Omega_{0}}\left(\ln \left[\frac{\gamma+\varepsilon}{\varepsilon}\right]\right)^{2} \mathrm{~d} x \\
& =\int_{\Omega_{0}}\left(\ln \left[\frac{\gamma+\varepsilon}{\gamma+\varepsilon-u^{+}}\right]\right)^{2} \mathrm{~d} x \\
& \leq\left\|w_{\varepsilon}\right\|_{L^{2}(\Omega)}^{2} \leq C \text { (independent of } \varepsilon \text { ) }
\end{aligned}
$$

This situation is impossible since $\left(\ln \left[\frac{\gamma+\varepsilon}{\varepsilon}\right]\right)^{2}$ converges towards $+\infty$ if $\varepsilon$ converges to $0^{+}$. Thus, $\gamma \leq 0$ and by definition of $\gamma, u \leq 0$ a.e. in $\Omega$.

The last step represents the conclusion. It is enough to notice, because of the linear character of the homogeneous equation, that if $u$ is a solution, $-u$ is also a solution. Thus $u \leq 0$ and $u \geq 0$, i.e. $u=0$.

Thus, $f=\bar{p}$ in (39) leads to the uniqueness of the solution to the primal problem, as it was presented in the study of the first case.

\section{The Case of dimension 3: Control of $p_{0}$}

It is possible to give the same study for a domain of $\mathbb{R}^{3}$ of the shape

$$
\Omega=\left\{(x, y, z) \in \mathbb{R}^{3}, \alpha<x<\beta, \varsigma<y<\tau, \gamma_{1}(x, y)<z<\gamma_{2}(x, y)\right\},
$$

where $\gamma_{i}$ are Lipschitzian functions. Problems are concentrated around the possibility of achieving the technique of uniqueness. In particular, the treatment of $\int_{\Omega} w \vec{D}_{1}\left(\sigma_{2}\right) \overrightarrow{\nabla \gamma} \mathrm{d} x$ when $w \in V^{0}$. That is to find again the result of Proposition 3.3 and more exactly, to know (for technical reasons) if $p_{0}>3$ is possible.

If one resumes the demonstration of this result, such as proposed in [19], one looks, page 46, for a value of $p_{0}>2$, close to 2 , such as, according to the notations of the context: $A_{q}^{1-\alpha\left(p_{0}\right)} \frac{\lambda_{2}-\lambda_{1}}{\lambda_{2}+\lambda_{1}} n^{\frac{1}{2}-\frac{1}{p_{0}}} \leq 1$.

Another method consists, for a fixed value of $p_{0}\left(p_{0}>3\right.$ for example), to suppose that $\lambda_{1}$ is close to $\lambda_{2}$. That is, the conditioning of the matrice $\left(a_{i j}\right)_{i j}$, for the matrix norm subordinate to the euclidian norm, is close to 1 .

However, in practice, the relative value of coefficients $\lambda_{s}$ and $\lambda_{a}$ which specify Kozeny-Carman's tensor of permeability in relation (8) limits the reach of this theoretical remark. The control of $p_{0}$, according to the spirit of the method, requires that the elliptic problem with symmetric and bounded coefficients is a "small" disturbance of Poisson's equation. What is not always turned out (case of strong anisotropy). As soon as the studied environment gives an importance to a space direction (in this particular case, the models of sedimentary basins enters in this frame via the hypothesis of vertical line compaction), the control of $p_{0}$ is very checked. In practice, $p_{0}$ remain very close to $2^{+}(c f .[4])$.

Acknowledgements. The authors wish to thank the referee for providing many interesting references in this topic and for very attentive and careful revision of the paper.

\section{REFERENCES}

[1] S.N. Antontsev and A.V. Domansky, Uniqueness generalizated solutions of degenerate problem in two-phase filtration. Numerical methods mechanics in continuum medium. Collection Sciences Research, Sbornik, t. 15, No. 6 (1984) 15-28 (in Russian).

[2] L. Badea, Adaptive mesh finite element method for the sedimentary basin problem. In honour of Academician Nicolae Dan Cristescu on his 70th birthday, Rev. Roumaine Math. Pures Appl. 45 (2000), No. 2 (2001) 171-181.

[3] C. Bardos, Problèmes aux limites pour les équations aux dérivées partielles partielles du premier ordre à coefficients réels. Ann. Sci. École Norm. Sup. 3 (1970) 185-233.

[4] A. Bensoussan, J.L. Lions and G. Papanicolaou, Asymptotic analysis for periodic structures. North-holland, Amsterdam (1978).

[5] P.A. Bourque, http://www.ggl.ulaval.ca/personnel/bourque/intro.pt/science.terre.html. 
[6] H. Brezis, Analyse fonctionnelle - Théorie et applications. Masson, Paris (1983).

[7] P.G. Ciarlet and J.L. Lions, Handbook of Numerical Analysis. Vol. II, Finite Element Methods (Part 1). North Holland (1991).

[8] A.C. Fowler and X. Yang, Fast and slow compaction in sedimentary basins. SIAM J. Appl. Math. 59 (1999) 365-385.

[9] G. Gagneux, Sur l'analyse de modèles de la filtration diphasique en milieu poreux, in Équations aux dérivées partielles et applications : Articles dédiés à J.L. Lions. Gauthier-Villars, Elsevier (1998) 527-540.

[10] G. Gagneux and M. Madaune-Tort, Analyse mathématique de modèles non linéaires de l'ingénierie pétrolière, Mathématiques \& Applications No. 22. Springer-Verlag (1996).

[11] G. Gagneux, A. Plouvier-Debaigt and G. Vallet, Modélisation et analyse mathématique d'un écoulement 2D monophasique dans un bassin sédimentaire faillé sous l'effet de la compaction verticale, Publication Interne du Laboratoire de Mathématiques Appliquées CNRS-ERS 2055, No. 2000-31 (2000).

[12] D. Gilbart and N.S. Trudinger, Elliptic Partial Differential Equations of Second Order. Springer-Verlag, Berlin (1977).

[13] G. Gödert and K. Hutter, Induced anisotropy in large ice shields: theory and its homogenization. Contin. Mech. Thermodyn. 10 (1998) 293-318.

[14] A.T. Ismail-Zade, A.I. Korotkii, B.M. Naimark and I.A. Tsepelev, Implementation of a three-dimensional hydrodynamic model for evolution of sedimentary basins. Comput. Math. Math. Phys. 38 (1998) 1138-1151.

[15] E. Ledoux, http://www.emse.fr/environnement/fiches/1_2_2.html.

[16] J.L. Lions, Quelques méthodes de résolution des problèmes aux limites non linéaires. Dunod, Paris (1969).

[17] X. Luo, G. Vasseur, A. Pouya, V. Lamoureux-Var and A. Poliakov, Elastoplastic deformation of porous media applied to the modelling of compaction at basin scale. Marine and Petroleum Geology 15 (1998) 145-162.

[18] N.G. Meyers, An Lp-estimate for the gradient of solutions of second order elliptic divergence equations. Ann. Sci. Norm. Sup. Pisa Cl. Sci. 17 (1963) 189-206.

[19] J. Nečas, Écoulements de fluide, Compacité par entropie, Collection Recherche et Mathématiques Appliquées, No. 10. Masson (1989).

[20] H. Obelembia Adande, Contribution à l'étude de l'unicité pour des systèmes d'équations de conservation. Cas des écoulements diphasiques incompressibles en milieu poreux, Thèse de l'Université de Pau (1996).

[21] O.A. Oleı̆nik, Uniqueness and stability of the generalized solution of the Cauchy problem for a quasi-linear equation. Uspekhi Mat. Nauk 14 (1959) 165-170.

[22] L. Perez, Modélisation de la compaction dans les bassins sédimentaires : Influence d'un comportement mécanique tensoriel, Thèse de l'ENSAM (1998).

[23] F. Schneider and S. Wolf, Quantitative HC potential evaluation using 3D basin modelling application to Franklin structure, central Graben, North Sea. UK Marine and Petroleum Geology 17 (2000) 841-856.

[24] F. Schneider, S. Wolf, I. Faille and D. Pot, A 3D basin model for hydrocarbon potential evaluation: Application to Congo offshore. Oil and Gas Science and Technology 55 (2000) 3-12.

[25] G. Sciarra, F. Dell'Isola and K. Hutter, A solid-fluid mixture model allowing for solid dilatation under external pressure. Contin. Mech. Thermodyn. 13 (2001) 287-306.

[26] M. Wangen, Two-phase oil migration in compacting sedimentary basins modelled by the finite element method. Int. J. Numer. Anal. Methods Geomech. 21 (1997) 91-120.

[27] M. Wangen, B. Antonsen, B. Fossum and L.K. Alm, A model for compaction of sedimentary basins. Appl. Math. Modelling $14(1990) 506-517$.

[28] Z. Wu and J. Yin, Some properties of functions in $B V_{x}$ and their applications to the uniqueness of solutions for degenerate quasilinear parabolic equations. Northeast. Math. J. 5 (1989) 395-422.

[29] E. Zakarian and R. Glowinski, Domain decomposition methods applied to sedimentary basin modeling. Math. Comput. Modelling 30 (1999) 153-178. 